\title{
Density-dependent electron scattering in photoexcited GaAs
}

\author{
Mics, Zoltán; D'Angio, Andrea; Jensen, Søren A.; Bonn, Mischa; Turchinovich, Dmitry
}

Published in:

Proceedings of the 38th International Conference on Infrared, Millimeter and Terahertz Waves IRMMW-THz 2013

Link to article, DOI:

10.1109/IRMMW-THz.2013.6665922

Publication date:

2013

Link back to DTU Orbit

Citation (APA):

Mics, Z., D'Angio, A., Jensen, S. A., Bonn, M., \& Turchinovich, D. (2013). Density-dependent electron scattering in photoexcited GaAs. In Proceedings of the 38th International Conference on Infrared, Millimeter and Terahertz Waves IRMMW-THz 2013 IEEE. https://doi.org/10.1109/IRMMW-THz.2013.6665922

\section{General rights}

Copyright and moral rights for the publications made accessible in the public portal are retained by the authors and/or other copyright owners and it is a condition of accessing publications that users recognise and abide by the legal requirements associated with these rights.

- Users may download and print one copy of any publication from the public portal for the purpose of private study or research.

- You may not further distribute the material or use it for any profit-making activity or commercial gain

- You may freely distribute the URL identifying the publication in the public portal 


\title{
Density-dependent electron scattering in photoexcited GaAs
}

\author{
Zoltán Mics ${ }^{\mathrm{a}}$, Andrea D’Angio ${ }^{\mathrm{a}}$, Søren A. Jensen ${ }^{\mathrm{a}, \mathrm{b}}$, Mischa Bonn ${ }^{\mathrm{a}}$ and Dmitry Turchinovich ${ }^{\mathrm{a}, \mathrm{c}}$ \\ ${ }^{a}$ Max Planck Institute for Polymer Research, Ackermannweg 10, 55128 Mainz, Germany \\ ${ }^{b}$ FOM Institute AMOLF, Science Park 104, 1098 XG Amsterdam, The Netherlands \\ ${ }^{\mathrm{c} D T U}$ Fotonik, Technical University of Denmark, 2800 Lyngby, Denmark
}

\begin{abstract}
In a series of systematic optical pump - terahertz probe experiments we study the density-dependent electron scattering rate in photoexcited GaAs in a large range of carrier densities. The electron scattering time decreases by as much as a factor of 4, from 320 to $60 \mathrm{fs}$, as the electron density changes by 4 orders of magnitude, from $10^{15}$ to $10^{19} \mathrm{~cm}^{-3}$.
\end{abstract}

\section{INTRODUCTION}

GaAs is an archetypical semiconductor, often used as a reference in optical pump - THz probe spectroscopy experiments $^{1}$. The $\mathrm{THz}$ photoconductivity of GaAs is well described by the Drude model, with carrier density $N$ and electron momentum scattering rate $\tau_{s}$ as parameters. Here, we show that the electron momentum scattering rate in photoexcited GaAs is a highly dynamic parameter with dramatic dependence on the carrier density.

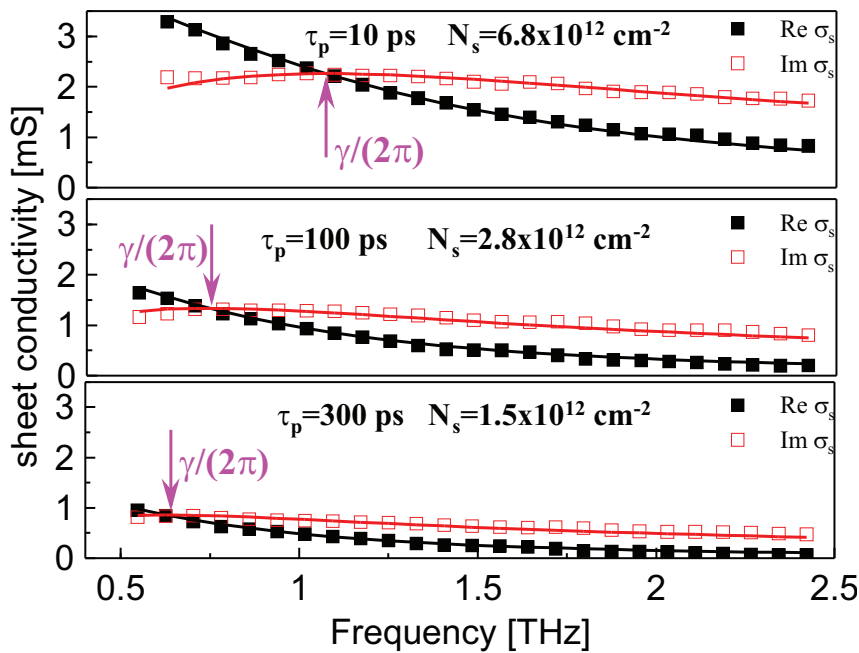

Figure 1: The measured sheet conductivity spectra (symbols) fitted by the Drude model (solid line) for selected pump-probe delays. The arrows show the change of the electron scattering time with the pump-probe delay.

\section{MEASUREMENT}

We investigated the carrier density - dependent scattering rate in GaAs in a systematic series of optical pump - $\mathrm{THz}$ probe measurements. As a sample, we used a semi-insulating GaAs wafer, photoexcited at $400 \mathrm{~nm}$ wavelength with variable pump fluence in the range of $1-200 \mu \mathrm{J} / \mathrm{cm}^{2}$ and THz-probed at selected times after photoexcitation in the range of 10-1000 ps. Our measurements directly yield the photoconductivity spectra of GaAs, which were found to be in excellent agreement with the Drude model. From the measured spectra the scattering rate of electrons and their sheet density was directly determined (Fig. 1.). In order to connect the calculated momentum scattering rates with the corresponding electron density, the volume of the electron-hole gas had to be determined. This quantity is not directly accessible from OPTP measurements in a strongly diffusive regime, as the spatial extent of the electron-hole gas in the material is a dynamic parameter.

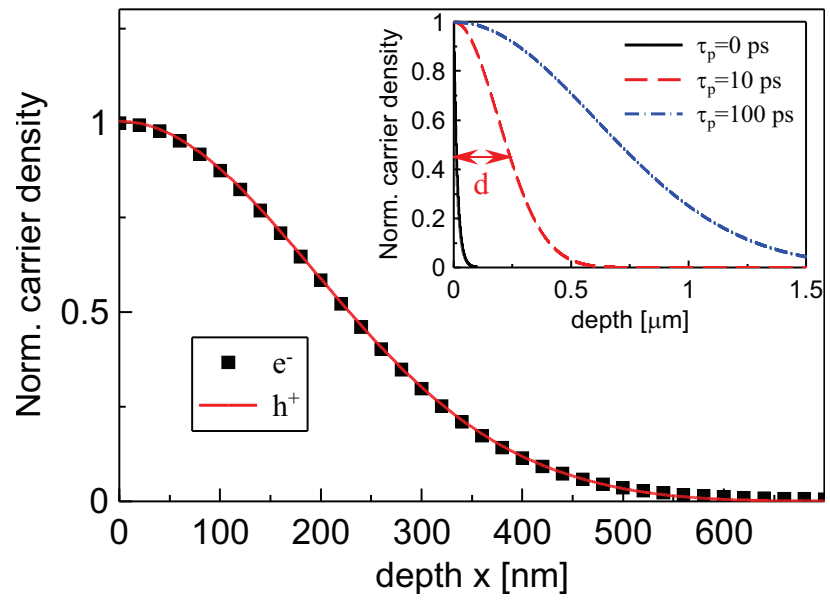

Figure 2: Numerical solution (at $t_{p}=10 \mathrm{ps}$ ) of the drift-diffusion equation illustrating the ambipolar diffusion, independent of pump fluence. Inset: The profile of electron concentration at selected pump-probe delays, and the effective electron gas width $d$.

\section{DATA ANALYSIS AND RESULTS}

In order to calculate the thickness of the electron-hole gas, we modeled the diffusion of electrons and holes into the unexcited regions of the semiconductor. Previous studies ${ }^{2}$ and our numerical simulations showed that this diffusion is ambipolar and essentially governed by the holes due to their lower mobility.

We used a simple diffusion model to simulate the expansion of the electron-hole gas. The influence of possible recombination and trapping on the shape of the spatial distribution of the electron-hole plasma was neglected. The ambipolar diffusion constant was calculated using the Einstein relation and from the fact the diffusion is driven by holes:

$$
D=\frac{2 k_{B} T}{\mu_{p}}
$$

where $\mathrm{T}$ is the temperature, and $\mu_{\mathrm{p}}=360 \mathrm{~cm}^{2} \mathrm{~V}^{-1} \mathrm{~s}$ is the hole mobility ${ }^{1,3}$. The initial thickness of the plasma (pump-probe delay $t_{p}=0$ ) is equal to the skin depth of GaAs at $400 \mathrm{~nm} d_{0}=15$ $\mathrm{nm}$. This thickness is negligible compared to the diffusion depth of the plasma in 10 ps (see the inset of Fig. 2), therefore we approximate the initial distribution of the plasma with a $\delta$ function. The distribution of the plasma at all times after photoexcitation is then described by a Gaussian function with effective thickness:

$$
d\left(t_{p}\right)=\sqrt{\pi D t_{p}}
$$

Using the calculated thickness of the plasma, we obtained the 
effective electron density for all the measured sheet photoconductivities such as shown in Fig. 1. Finally, these electron densities were correlated with the corresponding values of electron scattering time for every pump-probe delay $t_{p}$ and all the pump fluences used in our experiments.

This dependency of electron momentum scattering on the carrier density, shown in Fig. 3, is the main result of our work. We found that the scattering time dramatically decreases with increasing carrier density, fully independent of the pump fluence used (see Fig. 3.). It decreases by as much as a factor of 4, from 320 fs down to $60 \mathrm{fs}$, as the carrier density increases in the range $5 \times 10^{14}$ to $8 \times 10^{18} \mathrm{~cm}^{-3}$. In particular, this indicates that the phonons generated by the cooling of the photoexcited electrons do not contribute significantly to electron scattering. In order to simplify the use of our findings in applications, we have fitted the measured dependency $\mathrm{N}\left(\tau_{\mathrm{s}}\right)$ using the CaugheyThomas relation ${ }^{5}$, with the fit parameters shown in Fig. 3.

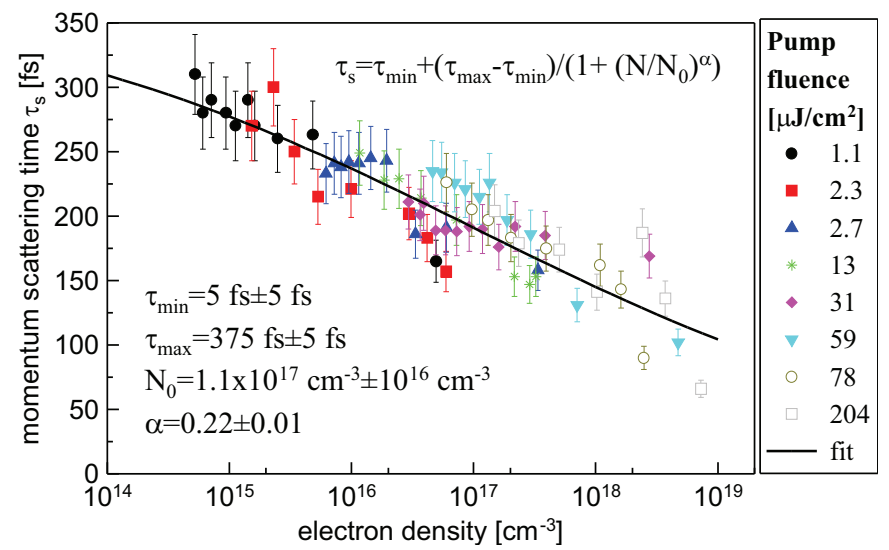

Figure 3: The electron momentum scattering time in GaAs as a function of carrier density. The data were fitted by the CaugheyThomas relation.

\section{REFERENCES}

[1] M. C. Beard, G. M. Turner and C. A. Schmuttenmaer, "Transient photoconductivity in GaAs as measured by time-resolved terahertz spectroscopy," Phys. Rev. B 62, 15764-15777 (2000)

[2] T. Dekorsy, H. Auer, H. J. Bakker, H. G. Roskos and H. Kurz, "THz electromagnetic emission by coherent infrared-active phonons", Phys. Rev. B 53, 4005-4014 (1996)

[3] B. A. Ruzicka, L. K. Werake, H. Samassekou and H. Zhao, "Ambipolar diffusion of photoexcited carriers in bulk GaAs", Appl. Phys. Lett. 97, 262119 (2010)

[4] Z. Mics, A. D'Angio, S. A. Jensen, M. Bonn and D. Turchinovich, "Density-dependent electron scattering in photoexcited GaAs in strongly diffusive regime", Appl. Phys. Lett. 102, 231120 (2013)

[5] D. M. Caughey and R. E. Thomas, "Carrier mobilities in silicon empirically related to doping and field", Proc. IEEE 55, 2192 (1967) 